\title{
Organic and elemental correlative imaging by using synchrotron light for illuminating molecular disorders in ALS relevant cells
}

Tanja Ducic ${ }^{1}$, Martin Kreuzer ${ }^{2}$, Si Chen ${ }^{3}$, Stefan Stamenkovic ${ }^{4}$ and Pavle Andjus ${ }^{4}$

${ }^{1}$ ALBA CELLS, Carrer de la Llum 2-26 | 08290 | Cerdanyola del Vallès| Barcelona | Spain, Barcelona, Spain, ${ }^{2}$ ALBA CELLS, Carrer de la Llum 2-26 | 08290 | Cerdanyola del Vallès| Barcelona | Spain, United States, ${ }^{3}$ Argonne National Laboratory, Lemont, Illinois, United States, ${ }^{4}$ Center for laser microscopy, Faculty of Biology, University of Belgrade, Serbia, United States

Principally, investigations on the molecular level in neuroscience, mainly demand a combination of novel techniques that would broaden the set of tools available for elucidation and study of cellular structural and elemental disease features [1]. Here we summarized a correlative approach of several synchrotron-based imaging and spectroscopy techniques we used for analyzing the subcellular changes and disorders in the neurodegenerative disease Amyotrophic Lateral Sclerosis (ALS). A set of familial types of ALS are caused by mutations in the metalloenzyme copper-zinc superoxide dismutase (SOD1). We employed different synchrotron radiation-based spectro-microscopies in situ, to investigate the astrocytes of the rat hSOD1 G93A ALS model that overexpresses human mutated SOD1 [2-5]. As in many neurodegenerative diseases, the imbalance of the redox-active trace metals, such as copper $(\mathrm{Cu})$, is associated with ALS [6,7]. The identification of $\mathrm{Cu}$ in situ in the cell, together with physiologically relevant macromolecules could support the understanding of processes involved in the pathological mechanism. Mutations in SOD1 contribute to the susceptibility of the protein to form cytoplasmic aggregates followed by the change of enzyme activity and the appearance of oxidative stress, intracellular perturbations such as endoplasmic reticulum and mitochondrial stress, along with the inhibition of axonal transport [8-10]. Alternatively, it was proposed that a loss of SOD1 function leads to superoxide $\left(\mathrm{O}_{2}^{-{ }^{-}}\right)$overproduction [11]. However, the relation of SOD1 mutations and ALS pathophysiology remains poorly understood (reviewed in [9]).

By using soft X-ray tomography, we analyzed protein aggregates along with alterations in the cellular ultrastructure, and elemental and organic composition by X-ray fluorescence microscopy (XRF) and Synchrotron Radiation based Fourier Transform Infrared micro-spectroscopy (SR-FTIR), respectively. These methods do not require invasive cellular preparation. In particular, we used cryo- preparation by vitrification for X-ray techniques and subsequently freeze-drying for FTIR, avoiding chemical embedding and cutting.

Our results demonstrate that large aggregates in the form of multivesicular inclusions form exclusively in the ALS model astrocytes [3]. Moreover, the cellular copper concentration and the amount of anti-parallel $\beta$-sheet structures were significantly changed within the cells of the ALS model $[3,4]$. Subsequently, we show that ALS cells contained more $\mathrm{Cu}$, which co-localized with total lipids, increased carbonyl groups and oxidized lipids, thus implying direct involvement of $\mathrm{Cu}$ in oxidative stress of lipidic components without direct connection to protein aggregation in situ [4]. Synchrotron light correlative methods link changes in lipids chemistry with the distribution of trace metals, connecting these potential biomarkers to an increased susceptibility to oxidative stress in single ALS astrocytes [4]. Recent efforts have been made to identify particular changes in lipid metabolism that could provide deeper insight into membrane rearrangements, that may serve as a robust biomarker for the disease (reviewed by [12]).

Figure 1 summarizes information obtained by synchrotron-based multivariate and correlative approach, employing several spectro-microscopical methods for the examination of ALS astrocyte cells [4]. This method may possibly be used for imaging of other specimens and we believe it has the potential to become an important tool for a more inclusive understanding of neurodegenerative disease progression at the sub-cellular level. 


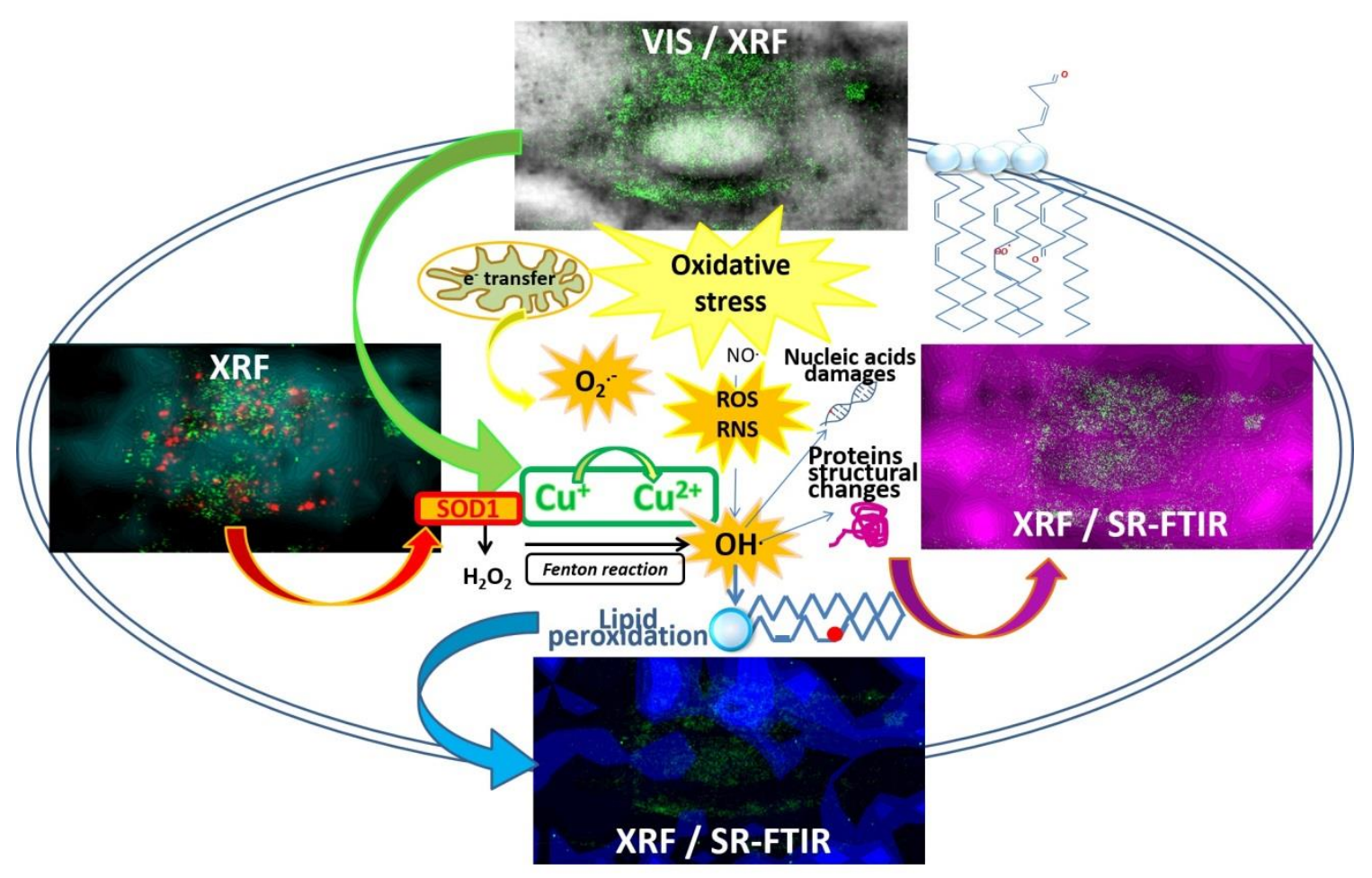

Figure 1. Schematic overview of simultaneous apply of synchrotron-based methods: Fourier-transform infrared micro-spectroscopy (SR-FTIR) and hard X-ray fluorescence microscopy (XRF) on ALS astrocyte cells (modified from [4]).

References

[1] T. Salditt, T. Dučić, Novel Opportunities by Coherent Optics, Humana Press, Totowa, NJ, (2014) 257-290. https://doi.org/10.1007/978-1-62703-983-3_11.

[2] S. Stamenković, T. Dučić, V. Stamenković, A. Kranz, P.R. Andjus, Neuroscience. 357 (2017) 37-55. https://doi.org/10.1016/j.neuroscience.2017.05.041.

[3] T. Dučić, S. Stamenković, B. Lai, P. Andjus, V. Lučić, Anal. Chem. 91 (2019) 1460-1471. https://doi.org/10.1021/acs.analchem.8b04273.

[4] M. Kreuzer, S. Stamenković, S. Chen, P. Andjus, T. Dučić, J. Biophotonics. 13 (2020). https://doi.org/10.1002/jbio.202000069.

[5] P. Andjus, S. Stamenković, T. Dučić, Eur. Biophys. J. 48 (2019). https://doi.org/10.1007/s00249-01901380-5.

[6] W.I.M. Vonk, L.W.J. Klomp, Biochem. Soc. Trans. 36 (2008) 1322-8. https://doi.org/10.1042/BST0361322.

[7] P.M. Roos, O. Vesterberg, T. Syversen, T.P. Flaten, M. Nordberg, Biol. Trace Elem. Res. 151 (2013) 159-70. https://doi.org/10.1007/s12011-012-9547-x.

[8] F. Navone, P. Genevini, N. Borgese, Cells. 4 (2015) 354-386. https://doi.org/10.3390/cells4030354.

[9] A. Vejux, A. Namsi, T. Nury, T. Moreau, G. Lizard, Front. Mol. Neurosci. 11 (2018) 12.

https://doi.org/10.3389/fnmol.2018.00012.

[10] Y. Hayashi, K. Homma, H. Ichijo, Adv. Biol. Regul. 60 (2016) 95-104.

https://doi.org/10.1016/j.jbior.2015.10.006.

[11] J. Beckman, J. Crow, (1993). Biochemical Society Transactions, 330, https://pdfs.semanticscholar.org/309f/e5b0207077cbad83a9e1f9dab841e7c9203f.pdf

[12] J.-L. González De Aguilar, Front. Neurol. 10 (2019) 284. https://doi.org/10.3389/fneur.2019.00284. 